\title{
BIPOLAR BAROTROPIC NON-NEWTONIAN COMPRESSIBLE FLUIDS
}

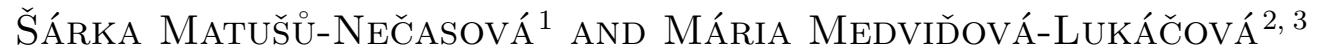

\begin{abstract}
We are interested in a barotropic motion of the non-Newtonian bipolar fluids . We consider a special case where the stress tensor is expressed in the form of potentials depending on $e_{i i}$ and $\left(\frac{\partial e_{i j}}{\partial x_{k}}\right)$. We prove the asymptotic stability of the rest state under the assumption of the regularity of the potential forces.
\end{abstract}

Mathematics Subject Classification. 76N10, 76A05, 35Q35.

Received: June 3, 1999. Revised: May 5, 2000.

\section{INTRODUCTION}

There are many substances which are capable of flowing but which exhibit flow characteristics that cannot be adequately described by the classical linearly viscous fluid model. In order to describe some of the departures from Newtonian behaviour (rheological properties, elastic features such as yield stress, stress relaxation and nonzero normal stress differences) many idealized material models have been suggested. During the last decades, mathematicians have also started to pay attention to these models and several results concerning the existence, uniqueness and stability of solutions have appeared, see [1-3, 5, 8-19,22-24, 26-28].

We will deal with compressible non-Newtonian fluids. The models which describe their rheological properties were studied from the mathematical point of view by Matušů-Nečasová, Lukáčová-Medvid’ová see [14,16]. They proved the existence and uniqueness of a weak solution. All of these results were studied on bounded domains. In the case of an isothermal process the stability of the rest state has been proved [16]. The global existence of equations of non-Newtonian compressible fluids when the coefficients of viscosity depend on the invariants of velocity field where the growth of these coefficients is not polynomial but exponential was proved in [12,13]. In the case of viscoelastic compressible fluids the existence of a classical solution of steady motion in the bounded domain was proved by Sy [27]. This result was extended to an exterior domain by Matušů-Nečasová et al. [15]. Both results are valid for small data only. One of the very interesting problem is the stability of the rest state. The crucial point of every proof of stability is the uniqueness of the steady state solutions. Beirão da

Keywords and phrases. Non-Newtonian compressible fluids, global existence, uniqueness, asymptotic stability, the rest state.

1 Mathematical Institute of the Academy of Sciences, Žitná 25, 11567 Prague 1, Czech Republic.

2 Institute of Analysis and Numerics, Otto-von-Guericke-Universität Magdeburg, Universitätsplatz 2, 39106 Magdeburg, Germany.

3 Institute of Mathematics, Faculty of Mechanical Engineering, Technical University of Brno, Technická 2, 61639 Brno, Czech Republic. 
Veiga [4] obtained a necessary and sufficient condition for the existence of a strictly positive solution of the following problem

$$
\begin{aligned}
& \partial_{i} p(\rho)=\rho \partial_{i} \xi_{i}, \quad i=1, \ldots, d \\
& \rho>0, \int_{\Omega} \rho(x) \mathrm{d} x=m \\
& v=0,
\end{aligned}
$$

where $\rho$ is the density, $m>0$ the total mass conserved by the flow, $p(\rho)$ is the pressure, $\xi$ is the potential of external forces which is locally Lipschitz on $\Omega, v$ is the velocity. It is easy to show that such a solution is necessarily unique. On the other hand, this restriction excludes a class of solutions with vacuum state $(\rho=0)$. The optimal condition for the solution of (1.1) to be unique is shown by Feireisl and Petzeltová $[6,7]$.

Here, our objective is to study the stability of the rest state of the barotropic motion. This model will be mathematically formulated in Chapter 1 . Chapter 2 is devoted to mathematical preliminaries and known results. In Chapter 3 we will prove the asymptotic stability of the rest state.

\section{Formulation of the Problem}

The barotropic motion of the bipolar non-Newtonial fluids is governed by the following system of the equations

$$
\begin{aligned}
\frac{\partial \rho}{\partial t}+\frac{\partial}{\partial x_{i}}\left(\rho v_{i}\right) & =0 \\
\frac{\partial}{\partial t}\left(\rho v_{i}\right)+\frac{\partial}{\partial x_{j}}\left(\rho v_{i} v_{j}\right)-\frac{\partial}{\partial x_{j}} \tau_{i j}^{V}(v) & =-\frac{\partial p}{\partial x_{i}}+\rho b_{i}, i=1,2, \ldots, d,
\end{aligned}
$$

where $\rho$ denotes the density, $v=\left(v_{1}, \ldots, v_{d}\right)$ is the velocity vector, $b=\left(b_{1}, \ldots, b_{d}\right)$ denotes the density of external forces and $p=p(\rho)$ is the pressure. We assume that $p \in C^{2}(0,+\infty)$. The equations (1.2), (1.3) are solved in the time-space cylinder $Q_{T}:=I \times \Omega, I=(0, T)$, where $\Omega \subset \mathbb{R}^{d}$ is a bounded domain with a smooth infinitely differentiable boundary $\partial \Omega$. Let us define

$$
\begin{aligned}
& P(\rho)=\rho \int_{1}^{\rho} \frac{p(\sigma)}{\sigma^{2}}, \quad \rho>0, \\
& P(0)=\lim _{\rho \rightarrow 0_{+}} P(\rho) .
\end{aligned}
$$

It may be verified (see [21]) that

$$
\begin{gathered}
\rho P^{\prime}(\rho)-P(\rho)=p(\rho), \quad \rho>0, \\
P^{\prime \prime}(\rho)=\frac{p^{\prime}(\rho)}{\rho}, \quad \rho>0, \\
P(\rho) \geq-k_{1}, \quad \rho \geq 0 .
\end{gathered}
$$

We suppose that the body forces are given and satisfy

$$
b \in L^{\infty}\left(Q_{T}\right)
$$


Now, we can specify the assumptions on the stress tensor $\tau_{i j}^{V}$. Namely:

$$
\begin{gathered}
\tau_{i j}^{V}=\frac{\partial V(\operatorname{Tr} e)}{\partial e_{i i}} \delta_{i j}-\frac{\partial}{\partial x_{k}}\left(\frac{\partial W(D e)}{\partial\left(\frac{\partial e_{i j}}{\partial x_{k}}\right)}\right) \\
D e=\left(\frac{\partial e_{i j}}{\partial x_{k}}\right)_{i, j, k=1}^{d}, \quad e=\left(e_{i j}\right)_{i, j=1}^{d}, \quad e_{i j}=\frac{1}{2}\left(\frac{\partial v_{i}}{\partial x_{j}}+\frac{\partial v_{j}}{\partial x_{i}}\right), \quad \operatorname{Tr} e=\left(e_{i i}\right)_{i=1}^{d} .
\end{gathered}
$$

Also, we consider the third stress tensor $\tau_{i j k}^{V}$, which has the following form

$$
\tau_{i j k}^{V}=\frac{\partial W(D e)}{\partial\left(\frac{\partial e_{i j}}{\partial x_{k}}\right)}
$$

Moreover, we will assume that the potentials $V, W$ satisfy the following conditions:

$$
\begin{aligned}
C_{1}(1+|D e|)^{q-2}|\xi|^{2} & \leq \frac{\partial^{2} W(D e)}{\partial\left(\frac{\partial e_{i j}}{\partial x_{k}}\right) \partial\left(\frac{\partial e_{i_{1} j_{1}}}{\partial x_{k_{1}}}\right)} \xi_{i j}^{k} \xi_{i_{1} j_{1}}^{k_{1}} \\
& \leq C_{2}(1+|D e|)^{q-2}|\xi|^{2} \\
C_{3}(1+|\operatorname{Tr} e|)^{q-2}|\xi|^{2} & \leq \frac{\partial^{2} V(\operatorname{Tr} e)}{\partial e_{i i} \partial e_{i_{1} i_{1}}} \xi_{i i} \xi_{i_{1} i_{1}} \\
& \leq C_{4}(1+|\operatorname{Tr} e|)^{q-2}|\xi|^{2}
\end{aligned}
$$

where $C_{1}, C_{2}, C_{3}, C_{4}$ are positive constants, $q>d$, and $|\cdot|$ is the usual Euclidean norm of a vector. Let

$$
\begin{gathered}
W(0)=0, V(0)=0 \\
\frac{\partial W}{\partial\left(\frac{\partial e_{i j}}{\partial x_{k}}\right)}(0)=0, \frac{\partial V}{\partial e_{i i}}(0)=0 .
\end{gathered}
$$

The system (1.2), (1.3) is completed by the initial conditions

$$
v(0)=v_{0}, \rho(0)=\rho_{0}, \rho_{0}>0 \quad \text { in } \Omega ;
$$

and the boundary conditions

$$
\tau_{i j k}^{V} \nu_{j} \nu_{k}=0 \quad \text { on }(0, T) \times \partial \Omega
$$

$(\nu$ is an outer normal to $\partial \Omega$ ) ,

$$
v=0 \quad \text { on }(0, T) \times \partial \Omega
$$




\section{Mathematical PRELiminaries}

By $L^{p}(\Omega)$ and $W^{l, p}(\Omega), 0 \leq p, l<\infty$, we denote the Lebesgue and Sobolev spaces, respectively, equipped with the standard norm.

First, we give the well-known results on the equilibrium solution, where we do not consider the cavitation of density. In the work of Beirão da Veiga [4] the necessary and sufficient conditions for the existence of the equilibrium solutions for an arbitrary $b \in L^{\infty}(\Omega)$ was proved. Let $p$ be a continuously differentiable real function defined on $\mathbb{R}^{+}=\{s \in R: s>0\}$, such that $p^{\prime}(s)>0, \forall s \in \mathbb{R}^{+}$. Let

$$
0<\operatorname{ess} \inf _{x \in \Omega} \rho(x), \text { ess } \sup _{x \in \Omega} \rho(x)<+\infty
$$

and

$$
\frac{1}{|\Omega|} \int_{\Omega} \rho(x) \mathrm{d} x=m
$$

for a fixed $m>0$, we define

$$
\pi(s)=\int_{0}^{s} \frac{p^{\prime}(t)}{t} \mathrm{~d} t, \quad \forall s \in \mathbb{R}^{+} .
$$

We denote $(a, f)$ the range of $\pi,(a, f)=\pi\left(\mathbb{R}^{+}\right)$.

Let us define $\phi=\pi^{-1}$. Clearly, $\phi((a, f))=\mathbb{R}^{+}$. Put $\phi(a)=0, \phi(f)=+\infty$.

Definition 2.1. Let $b \in L^{\infty}(\Omega)$. A function $\rho$ is called an equilibrium solution of (1.1) if $\rho \in L^{\infty}(\Omega)$ and if

$$
\pi(\rho(x))=\xi(x)+c \quad \text { a.e. in } \Omega
$$

and (2.1), (2.2) hold.

We set $n_{o}=\operatorname{ess} \inf b$ in $\Omega, N_{0}=\operatorname{ess} \sup b$ in $\Omega$.

Theorem 2.1. Let $b \in L^{\infty}(\Omega)$ be given. There exists an equilibrium solution $\rho(x)$ if and only if there exists a constant

$$
c \in\left(a-n_{0}, f-N_{0}\right),
$$

such that

$$
\frac{1}{|\Omega|} \int_{\Omega} \phi(c+\xi(x)) \mathrm{d} x=m .
$$

If such a constant exists then the (unique) equilibrium solution is given by

$$
\rho(x)=\phi(c+\xi(x)), \forall x \in \Omega .
$$

Proof. see [4].

Theorem 2.2. Under the assumptions of Theorem 2.1 there exists an equilibrium solution $\rho(x)$ if and only if

$$
a-n_{0}<f-N_{0}
$$


and

$$
\frac{1}{|\Omega|} \int_{\infty} \phi\left(a-n_{0}+\xi(x)\right) \mathrm{d} x<m<\frac{1}{|\Omega|} \phi\left(f-N_{0}+\xi(x)\right) \mathrm{d} x .
$$

In this case the equilibrium solution $\rho(x)$ is given by (2.6), where $c$ is the (unique) solution of (2.4)-(2.5).

Proof. see [4].

We mention a weak formulation of the problem (1.2), (1.3), (1.12)-(1.14).

Definition 2.2. A pair $(\rho, v)$ is said to be a weak solution of the problem (1.2), (1.3), (1.12)-(1.14), if the following conditions are satisfied

(i) $\quad \rho \in L^{\infty}\left(I ; W^{1, q}(\Omega)\right)$,

(ii) $\quad \frac{\partial \rho}{\partial t} \in L^{\infty}\left(I ; L^{q}(\Omega)\right)$,

(iii) $\quad v \in L^{\infty}\left(I ; W^{2, q}(\Omega) \cap W_{0}^{1,2}(\Omega)\right)$,

(iv) $\quad \frac{\partial v}{\partial t} \in L^{2}\left(Q_{T}\right)$

(v) the continuity equation (1.2) is satisfied in the sense of distributions on $Q_{T}$,

$$
\int_{\Omega} \frac{\partial}{\partial t}\left(\rho v_{i}\right) \varphi_{i}-\int_{\Omega} \rho v_{i} v_{j} \frac{\partial \varphi_{i}}{\partial x_{j}}-\int_{\Omega} p(\rho) \frac{\partial \varphi_{i}}{\partial x_{i}}+\int_{\Omega} \frac{\partial V}{\partial e_{i i}}(\operatorname{Tr} e(v)) e_{i i}(\varphi) \delta_{i j}+\frac{\partial W(D e(v))}{\partial\left(\frac{\partial e_{i j}}{\partial x_{k}}\right)} \frac{\partial e_{i j}}{\partial x_{k}}(\varphi)=\int_{\Omega} \rho b_{i} \varphi_{i}
$$
holds for a.e. $t \in I$ and for every $\varphi=\left(\varphi_{1}, \ldots, \varphi_{d}\right) \in W^{2, q}(\Omega) \cap W_{0}^{1,2}(\Omega)$,

(vii) the initial conditions (1.12) with $\rho_{0} \in C^{1}(\bar{\Omega})$ and $v_{0} \in W^{2, q}(\Omega) \cap W_{0}^{1,2}(\Omega)$ are fulfilled.

In [14] authors proved the existence and uniqueness of weak solution of the similar problem except the nonlinear potential $V$, which depends on the whole tensor $e=\left(e_{i j}\right)_{i, j=1}^{d}$ instead of $\operatorname{Tr} e=\left(e_{i i}\right)_{i=1}^{d}$ as in (1.5). Nevertheless the existence and uniqueness can be proved in the same way. In what follows we only point out the parts which differ from results stated in [14].

Theorem 2.3. (Existence of a weak solution).

Let $\rho_{0} \in C^{1}(\bar{\Omega}), \rho_{0}>0$ in $\bar{\Omega}$, and $v_{0} \in W^{2, q}(\Omega) \cap W_{0}^{1,2}(\Omega)$. Let the assumptions (1.8)-(1.11) hold. Then there is at least one weak solution $(\rho, v)$ to the problem $(1.2),(1.3),(1.12)-(1.14)$ such that

$$
\begin{aligned}
& \rho \in L^{\infty}\left(I ; W^{1, q}(\Omega)\right), \frac{\partial \rho}{\partial t} \in L^{\infty}\left(I ; L^{q}(\Omega)\right), \\
& v \in L^{\infty}\left(I ; W^{2, q}(\Omega) \cap W_{0}^{1,2}(\Omega)\right), \frac{\partial v}{\partial t} \in L^{2}\left(Q_{T}\right) .
\end{aligned}
$$

Proof. First step is based on the modified Galerkin method and the method of characteristics, which give us the following identities see [14]:

$$
\begin{gathered}
\int_{\Omega_{t}} \rho_{m} \mathrm{~d} x=\int_{\Omega} \rho_{0} \mathrm{~d} x=m_{0} \\
\frac{1}{2} \int_{Q_{T}} \frac{\partial}{\partial t}\left(\rho_{m}\left|v^{m}\right|^{2}\right)+\int_{Q_{T}} \frac{\partial}{\partial t}\left(P\left(\rho_{m}\right)\right)+\int_{Q_{T}} \frac{\partial V}{\partial e_{i i}}\left(\operatorname{Tr} e\left(v^{m}\right)\right) e_{i i}\left(v^{m}\right)+\frac{\partial W}{\partial\left(\frac{e_{i j}}{\partial x_{k}}\right)} \frac{\partial e_{i j}}{\partial x_{k}}\left(v^{m}\right)=\int_{Q_{T}} \rho_{m} b_{i} v_{i}^{m}, \\
\int_{Q_{T}} \rho_{m}\left(\frac{\partial v_{i}^{m}}{\partial t}\right)^{2}+\int_{Q_{T}} \rho_{m} \frac{\partial v_{i}^{m}}{\partial x_{l}} v_{j}^{m} \frac{\partial v_{i}^{m}}{\partial t}+\int_{\Omega_{t}} V\left(\operatorname{Tr} e\left(v^{m}\right)\right)+W\left(D e\left(v^{m}\right)\right)+\int_{Q_{T}} \frac{\partial p\left(\rho_{m}\right)}{\partial x_{i}} \frac{\partial v_{i}^{m}}{\partial t}= \\
\int_{\Omega_{0}} V\left(\operatorname{Tr} e\left(v^{m}(0)\right)\right)+W\left(D e\left(v^{m}(0)\right)\right)+\int_{Q_{T}} \rho_{m} b_{i} \frac{\partial v_{i}^{m}}{\partial t} .
\end{gathered}
$$


In the second step the limiting process is performed. We mention only the convergence in the nonlinear term which is different now and also was not clearly explained in [14]. It reads

$$
\int_{\Omega} \frac{\partial V}{\partial \operatorname{Tr} e}\left(\operatorname{Tr} e\left(v^{m}\right)\right) e_{i i}\left(v^{m}\right)+\frac{\partial W}{\partial\left(\frac{\partial e_{i j}}{\partial x_{k}}\right)}\left(D e\left(v^{m}\right)\right) \frac{\partial e_{i j}\left(v^{m}\right)}{\partial x_{k}} .
$$

Let us define

$$
G_{1}\left(v^{m}\right)=\frac{\partial V}{\partial \operatorname{Tr} e}\left(\operatorname{Tr} e\left(v^{m}\right)\right) \operatorname{Tr} e\left(v^{m}\right)
$$

and

$$
G_{2}\left(v^{m}\right)=\frac{\partial W}{\partial\left(\frac{\partial e_{i j}}{\partial x_{k}}\right)}\left(D e\left(v^{m}\right)\right) \frac{\partial e_{i j}\left(v^{m}\right)}{\partial x_{k}}
$$

We set

$$
X_{m}^{s}=\int_{0}^{s}\left(G_{1}\left(v^{m}\right)-G_{1}(\varphi), e\left(v^{m}\right)-e(\varphi)\right) \mathrm{d} t+\int_{0}^{s}\left(G_{2}\left(v^{m}\right)-G_{2}(\varphi), D e\left(v^{m}\right)-D e(\varphi)\right) \mathrm{d} t+\frac{1}{2}\left|\rho_{m} v^{m}(s)\right|^{2} .
$$

Choosing a subsequence $v_{n_{k}}$ which we denote as $v_{n}$ and using monotonicity of $X_{m}^{s}$ we obtain

$$
\liminf _{m \rightarrow+\infty} X_{m}^{s} \geq \frac{1}{2}|\rho v(s)|^{2} .
$$

Thus, it follows from (2.11) that

$$
\begin{aligned}
X_{m}^{s}= & \int_{0}^{s}\left(\rho_{m} b, v^{m}\right) \mathrm{d} t+\frac{1}{2}\left|\rho_{0 m} v_{0}^{m}\right|^{2}-\int_{0}^{s}\left(G_{2}\left(v^{m}\right), D e(\varphi)\right) \mathrm{d} t-\int_{0}^{s}\left(G_{2}(\varphi), D e\left(v^{m}-\varphi\right)\right) \mathrm{d} t \\
& -\int_{0}^{s}\left(G_{1}\left(v^{m}\right), e(\varphi)\right) \mathrm{d} t-\int_{0}^{s}\left(G_{1}(\varphi), e\left(v^{m}-\varphi\right)\right) \mathrm{d} t \\
& \rightarrow \int_{0}^{s}(\rho b, v)+\frac{1}{2}\left|\rho_{0} v_{0}\right|^{2}-\int_{0}^{s}\left(\xi_{1}, D e(\varphi)\right)-\int_{0}^{s}\left(G_{2}(\varphi), D e(v-\varphi)\right)-\int_{0}^{s}\left(\xi_{2}, e(\varphi)\right) \\
& -\int_{0}^{s}\left(G_{1}(\varphi), e(v-\varphi)\right) \geq \frac{1}{2}|\rho v(s)|^{2} .
\end{aligned}
$$

Finally, we get

$$
\int_{0}^{t}\left(\xi_{1}-G_{2}(\varphi), D e(v)-D e(\varphi)\right)+\left(\xi_{2}-G_{1}(\varphi), e(v-\varphi)\right) \geq 0
$$

which implies that

$$
\xi_{1}=G_{2}(v), \quad \xi_{2}=G_{1}(v)
$$

This concludes the proof.

Theorem 2.4. (Uniqueness of the weak solution).

Let the assumptions of Theorem 2.3 be fulfilled. Then the weak solution obtained in Theorem 2.3 is unique.

Proof. Is analogous to the proof of uniqueness in [14]. 


\section{UnCONDItIONAL ASYMPtotic STABILITy OF THE REST STATE}

The goal of this section is to prove the stability of the rest state $(\rho, 0)$, characterized by $(1.1)$. Let $b=\frac{\partial \xi}{\partial x_{i}}$ with $\xi \in W^{2, \infty}(\Omega), \frac{\mathrm{d} p}{\mathrm{~d} \rho}>0$ and let $\left(\rho_{0}, v_{0}\right)$ satisfy conditions $\rho_{0} \in C^{1}(\bar{\Omega}), \rho_{0}>0$ in $\bar{\Omega}, v_{0} \in W^{2, q}(\Omega) \cap W_{0}^{1,2}(\Omega)$.We specify the class of perturbated flows where the rest state will be stable:

$$
\begin{aligned}
\mathcal{J}= & \left\{(\tilde{\rho}, \tilde{v}): \tilde{\rho} \in L^{\infty}\left(I, W^{1, q}(\Omega)\right), \tilde{v} \in L^{\infty}\left(I, W^{2, q}(\Omega)\right) \cap W_{0}^{1,2}(\Omega)\right), \\
& \frac{\partial \tilde{\rho}}{\partial t} \in L^{\infty}\left(I, L^{q}(\Omega)\right), \frac{\partial \tilde{v}}{\partial t} \in L^{2}\left(Q_{T}\right), \text { for any } \mathrm{T}, \text { and there exist } \\
& \delta_{1}, \delta_{2} \text { such that }, 0<\delta_{1} \leq \tilde{\rho}<\delta_{2} \text { uniformly in }(0, \infty) \times \bar{\Omega},
\end{aligned}
$$$$
(\tilde{\rho}, \tilde{v}) \text { is a weak solution of }(1.2),(1.3)\} \text {. }
$$

Multiplying (1.3) by $v$ we obtain

$$
\frac{1}{2} \frac{\mathrm{d}}{\mathrm{d} t} \int_{\Omega}\left[\rho|v|^{2}+\mathcal{P}(\rho)+\xi \rho\right] \mathrm{d} x+\|u\|_{W^{2, q}}=0
$$

Let us denote $\sigma(t)=\left(\int_{t-1}^{t}\|v\|_{W^{2, q}(\Omega)}^{q}\right)^{1 / q}$. From (3.1) we have that

$$
\lim _{t \rightarrow \infty} \sigma(t)=0
$$

Moreover, we would like to prove that

$$
v(t) \rightarrow 0 \quad \text { strongly in } L^{2}(\Omega) \text { as } t \rightarrow \infty \text {. }
$$

It holds

$$
\frac{1}{2} \frac{\mathrm{d}}{\mathrm{d} t} \int_{\Omega} \rho|v|^{2}+\|v\|_{W^{2, q}(\Omega)}+\|\nabla \cdot v\|_{L^{q}(\Omega)}-\int_{\Omega} p(\rho) \nabla \cdot v=\int_{\Omega} \rho b v \mathrm{~d} x .
$$

By integrating the above relation with respect to $t$ from $s$ to $\mathrm{t}, 0 \leq s \leq t<\infty$ and with respect to $s$ from $t-1$ to $t$ we obtain

$$
\begin{aligned}
\frac{1}{2}\|\sqrt{\rho(t)} v(t)\|_{L^{2}(\Omega)}^{2}+\int_{t-1}^{t} \int_{s}^{t}\|v\|_{W^{2, q}(\Omega)} & +\int_{t-1}^{t} \int_{s}^{t}\|\nabla \cdot v\|_{L^{q}(\Omega)}= \\
& \frac{1}{2} \int_{t-1}^{t}\|\sqrt{\rho(t)} v(t)\|_{L^{2}(\Omega)}^{2}+\int_{t-1}^{t} \int_{s}^{t} \int_{\Omega}|\rho b v+p(\rho) \nabla \cdot v| \mathrm{d} x \mathrm{~d} \tau \mathrm{d} s
\end{aligned}
$$

Further,

$$
\begin{gathered}
\int_{t-1}^{t} \int_{s}^{t} \int_{\Omega}|\rho b v| \leq\|\rho\|_{L^{\infty}\left(0, T ; L^{q}(\Omega)\right)}\|b\|_{L^{\infty}\left(Q_{T}\right)}\left(\int_{t-1}^{t}\|v\|_{W^{2, q}(\Omega)}^{q} \mathrm{~d} \tau\right) \leq c\|b\|_{L^{\infty}\left(Q_{T}\right)} \sigma(t), \\
\int_{t-1}^{t} \int_{s}^{t} \int_{\Omega} p(\rho) \nabla \cdot v \leq c \sup _{\delta_{1} \leq r \leq \delta_{2}} p(r) \sigma(t) .
\end{gathered}
$$

Then

$$
\|\sqrt{\rho(t)} v(t)\|_{L^{2}(\Omega)}^{2} \leq c\left(1+\|b\|_{L^{\infty}\left(Q_{T}\right)}\right) \sigma(t) .
$$

From (3.2), using the fact that $\rho>0$, we get that

$$
v(t) \rightarrow 0 \quad \text { strongly in } L^{2}(\Omega) \text { as } t \rightarrow \infty \text {. }
$$


Now, we define a function $w(t)$ in $Q_{T}$ as the solution of the following problem

$$
\begin{aligned}
& \Delta w=\nabla \cdot(\widetilde{\rho} b) \quad \text { in } Q_{T}, \\
& \frac{\partial w}{\partial n}=\widetilde{\rho} b \cdot n \quad \text { on } \partial \Omega \times[0, \infty), \\
& \int_{\Omega} w \mathrm{~d} x=0 .
\end{aligned}
$$

The goal is to prove that

$$
\widetilde{\rho}(t) \rightarrow \rho \text { in } L^{2}(\Omega) \quad \text { as } t \rightarrow \infty
$$

where

$$
\begin{aligned}
& \nabla p(\rho)=\rho b \text { in } \Omega, \\
& \int_{\Omega} \rho \mathrm{d} x=\int_{\Omega} \rho_{0} \mathrm{~d} x .
\end{aligned}
$$

Firstly, we shall prove that

$$
\left|\int_{\Omega}[p(\tilde{\rho}(t))-w(t)](h-M h) \mathrm{d} x\right| \leq c \sigma(t)\|h\|_{L^{2}(\Omega)}, \quad h \in L^{2}(\Omega),
$$

with a constant $c$ independent of $w$. We set

$$
M h=|\Omega|^{-1} \int_{\Omega} h \mathrm{~d} x .
$$

For this purpose we shall firstly estimate the integral

$$
I(t)=\int_{t-1}^{t} \varphi(s-t) \int_{\Omega}[p(\tilde{\rho}(s))-w(s)] h \mathrm{~d} x \mathrm{~d} s, \quad \varphi \in C_{0}^{\infty}(-1,0),
$$

where $\varphi$ is a fixed function such that $\int_{-1}^{0} \varphi(\tau) \mathrm{d} \tau=1$.

Let $\psi$ be a solution to the following problem for arbitrary $h \in L^{2}(\Omega)$

$$
\begin{aligned}
& \Delta \psi=h-M h \quad \text { in } \Omega \\
& \frac{\partial \psi}{\partial n}=0 \quad \text { on } \partial \Omega \\
& \int_{\Omega} \psi \mathrm{d} x=0 .
\end{aligned}
$$

Applying the classical results on boundary-value problems for elliptic equations it yields

$$
\begin{aligned}
\|\psi\|_{W^{2,2}(\Omega)} & \leq c\|h-M h\|_{L^{2}(\Omega)}, \\
\|h-M h\|_{L^{2}(\Omega)} & \leq\|h\|_{L^{2}(\Omega)},
\end{aligned}
$$


with a constant $c>0$ independent of $h$. Substituting (3.9) into (3.8) we find

$$
\begin{aligned}
& I(t)=-\int_{t-1}^{t} \varphi(s-t) \int_{\Omega}[w(s)-p(\tilde{\rho}(s))] \Delta \psi \mathrm{d} x \mathrm{~d} s \\
& =\int_{t-1}^{t} \varphi(s-t) \int_{\Omega}[\tilde{\rho}(s) b-\nabla p(\tilde{\rho}(s))] \nabla \psi \mathrm{d} x \mathrm{~d} s \\
& =\int_{t-1}^{t} \varphi(s-t) \int_{\Omega}\left[\tilde{\rho} \frac{\partial v(s)}{\partial s}+\tilde{\rho}(s) v(s) \nabla v(s)-\operatorname{div} \tau_{i j}\right] \nabla \psi \mathrm{d} x \mathrm{~d} s \\
& =\int_{t-1}^{t} \varphi(s-t) \int_{\Omega} \frac{\partial \tilde{\rho} v}{\partial s} \nabla \psi+\int_{t-1}^{t} \varphi(s-t) \int_{\Omega} \nabla \cdot(\tilde{\rho}(s) v(s) v(s)) \nabla \psi \\
& +\int_{t-1}^{t} \varphi(s-t) \int_{\Omega} \tau_{i j} \nabla \nabla \psi \\
& =-\int_{t-1}^{t} \varphi^{\prime}(s-t) \int_{\Omega} \tilde{\rho}(s) v(s) \nabla \psi-\int_{t-1}^{t} \varphi(s-t) \int_{\Omega} \tilde{\rho}(s) v(s) v(s) \nabla \nabla \psi \\
& +\int_{t-1}^{t} \varphi(s-t) \int_{\Omega} \frac{\partial^{2} W}{\partial\left(\frac{\partial e_{i j}}{\partial x_{k}}\right) \partial\left(\frac{\partial e_{i j}}{\partial x_{l}}\right)}\left(\frac{\partial e_{i j}}{\partial x_{l}}\right) \nabla \nabla \psi+\int_{t-1}^{t} \varphi(s-t) \int_{\Omega} \frac{\partial V(\operatorname{Tr} e)}{\partial e_{i i}} \Delta \psi \\
& \leq \sup _{t \in(0, T)}\|\tilde{\rho}(t)\|_{L^{q}(\Omega)}\left(\int_{-1}^{0} \varphi^{\prime}(\tau)^{q^{\prime}} \mathrm{d} \tau\right)^{1 / q^{\prime}}\|\nabla \psi\|_{L^{2}(\Omega)}\left(\int_{t-1}^{t}\|v(s)\|_{W^{2, q}(\Omega)}^{q}\right)^{1 / q} \\
& +\sup _{t \in(0, T)}\|\tilde{\rho}(t)\|_{L^{q}(\Omega)}\left(\int_{-1}^{0} \varphi(\tau)^{q^{\prime}} \mathrm{d} \tau\right)^{1 / q^{\prime}}\|\psi\|_{W^{2,2}(\Omega)}\left(\int_{t-1}^{t}\|v(s)\|_{W^{2, q}(\Omega)}^{q}\right)^{2 / q} \\
& +\left(\int_{-1}^{0} \varphi(\tau)^{q^{\prime}} \mathrm{d} \tau\right)^{1 / q^{\prime}}\|\psi\|_{W^{2,2}(\Omega)}\left(\int_{t-1}^{t}\|v\|_{W^{2, q}(\Omega)}^{q}\right)^{1 / q} \\
& +\left(\int_{-1}^{0}\|\varphi(\tau)\|^{q^{\prime}} \mathrm{d} \tau\right)^{1 / q^{\prime}}\left(\int_{0}^{t}\|v\|_{W^{2, q}(\Omega)}^{q}\right)^{1 / q}\|h\|_{L^{2}(\Omega)} \leq\|\varphi\|_{1, q^{\prime}}\|h\|_{L^{2}(\Omega)} \sigma(t) .
\end{aligned}
$$

However, $\varphi$ is an arbitrary fixed function, which implies that

$$
I(t) \leq c \sigma(t)\|h\|_{L^{2}(\Omega)}, \quad t \geq 0
$$

Now, we go back and prove (3.7).

$$
\begin{aligned}
\left|\int_{\Omega}[p(\tilde{\rho}(t))-w(t)](h-M h) \mathrm{d} x\right|= & \left|\int_{t-1}^{t} \varphi(s-t) \int_{\Omega}(\nabla p(\tilde{\rho}(t))-\tilde{\rho}(t) b) \nabla \psi \mathrm{d} x \mathrm{~d} s\right| \\
\leq & |I(t)|+\left|\int_{t-1}^{t} \varphi(s-t) \int_{\Omega}[p(\tilde{\rho}(t))-p(\tilde{\rho}(s))][h-M h] \mathrm{d} x \mathrm{~d} s\right| \\
& +\left|\int_{t-1}^{t} \varphi(s-t) \int_{\Omega}[\tilde{\rho}(t)-\tilde{\rho}(s)] b \nabla \psi \mathrm{d} x \mathrm{~d} s\right| .
\end{aligned}
$$

We have

$$
\tilde{\rho}(t)-\tilde{\rho}(s)=\int_{s}^{t} \frac{\partial \tilde{\rho}}{\partial \tau} \mathrm{d} \tau=-\int_{s}^{t} \nabla \cdot(\tilde{\rho}(\tau) u(\tau)) \mathrm{d} \tau
$$


Hence

$$
\begin{aligned}
\left|\int_{t-1}^{t} \varphi(s-t) \int_{\Omega}[\tilde{\rho}(t)-\tilde{\rho}(s)] b \cdot \nabla \psi\right|= & \left|\int_{t-1}^{t} \int_{s}^{t} \varphi(s-t) \int_{\Omega} \tilde{\rho}(\tau) u(\tau) \nabla(b \cdot \nabla \psi) \mathrm{d} x \mathrm{~d} \tau \mathrm{d} s\right| \\
\leq & \|\tilde{\rho}\|_{L^{\infty}\left(0, T ; L^{q}(\Omega)\right)}\|\varphi\|_{L^{q^{\prime}(0, T)}}\|\nabla b\|_{L^{\infty}\left(0, T ; L^{\infty}(\Omega)\right.}\|\nabla \psi\|_{L^{2}(\Omega)} \\
& +\|b\|_{L^{\infty}\left(0, T ; L^{\infty}(\Omega)\right.}\|\psi\|_{W^{2,2}(\Omega)}\left(\int_{t-1}^{t}\|v\|_{W^{2, q}(\Omega)}^{q} \mathrm{~d} s\right)^{1 / q} \\
\leq & c\|b\|_{W^{1, \infty}(\Omega)}\|\psi\|_{W^{2,2}(\Omega)} \sigma(t) .
\end{aligned}
$$

The second term of the RHS of (3.13) can be rewriten in the following form

$$
\int_{t-1}^{t} \varphi(s-t) \int_{\Omega}[p(\tilde{\rho}(t))-p(\tilde{\rho}(s))](h-M h) \mathrm{d} x \mathrm{~d} s=\int_{t-1}^{t} \varphi(s-t) \int_{\Omega} \int_{s}^{t} d_{\tau} p(\tilde{\rho}(\tau))(h-M h) \mathrm{d} \tau \mathrm{d} s \mathrm{~d} x .
$$

From the continuity equation it follows that

$$
\frac{\partial p(\tilde{\rho})}{\partial t}=-\left(v p^{\prime}(\tilde{\rho}) \nabla \tilde{\rho}+\tilde{\rho} p^{\prime}(\tilde{\rho}) \nabla \cdot v\right)
$$

Thus, we can estimate (3.16) from above

$$
\begin{aligned}
\left|\int_{t-1}^{t} \varphi(s-t) \int_{s}^{t} \int_{\Omega} d_{\tau} p(\tilde{\rho}(\tau))(h-M h) \mathrm{d} x \mathrm{~d} \tau \mathrm{d} s\right| \leq & \left|\int_{t-1}^{t} \varphi(s-t) \int_{s}^{t} \int_{\Omega} v(\tau) p^{\prime}(\tilde{\rho}(\tau)) \nabla \tilde{\rho}(\tau)(h-M h) \mathrm{d} x \mathrm{~d} \tau \mathrm{d} s\right| \\
& +\left|\int_{t-1}^{t} \varphi(s-t) \int_{s}^{t} \int_{\Omega} \tilde{\rho}(\tau) p^{\prime}(\tilde{\rho}) \nabla \cdot v(h-M h) \mathrm{d} x \mathrm{~d} \tau \mathrm{d} s\right| \\
\leq & \|\varphi\|_{L^{\infty}(0, T)} \sup _{\delta_{1} \leq r \leq \delta_{2}} p^{\prime}(r) \sigma(t)\left(\int_{t-1}^{t}\|\nabla \tilde{\rho}(\tau)\|_{L^{q}(\Omega)}^{q}\right)^{1 / q}\|h\|_{L^{2}(\Omega)} \\
& +c\|\varphi\|_{L^{q^{\prime}(0, T)} \sup _{\delta_{1} \leq r \leq \delta_{2}} r p^{\prime}(r)\|h\|_{L^{2}(\Omega)} \sigma(t) \leq c \sigma(t)\|h\|_{L^{2}(\Omega)},}
\end{aligned}
$$

which together with (3.12), (3.13) and (3.15) leads to (3.7). But it follows from (3.7) that

$$
p(\tilde{\rho}(t))-w(t)-M p(\tilde{\rho}(t)) \rightarrow 0 \quad \text { in } L^{2}(\Omega) \quad t \rightarrow \infty .
$$

In fact, there exists a subsequence $t_{n} \rightarrow \infty$ such that $\tilde{\rho}\left(t_{n}\right) \rightarrow \rho$ weakly in $L^{2}$, where $w(t)$ is a solution of (3.4) for any $t$. The sequence $w\left(t_{n}\right)$ is compact in $L^{2}(\Omega)$. Choosing a subsequence converging to $w_{\infty} \in L^{2}(\Omega)$ we find that $w_{\infty}=A(\rho b)$, where $A$ stands for the solution operator of (3.4). Thus, $p\left(\widetilde{\rho}\left(t_{n}\right)\right)-M p\left(\widetilde{\rho}\left(t_{n}\right)\right) \rightarrow$ $w_{\infty}$ in $L^{2}(\Omega)$ and a.e. in $\Omega$. Since $\widetilde{\rho}$ belongs to $\mathcal{J}$, we get that $M p\left(\tilde{\rho}\left(t_{n}\right)\right) \rightarrow p_{\infty}$. This yields

$$
\widetilde{\rho}\left(t_{n}\right) \rightarrow p^{-1}\left(w_{\infty}+p_{\infty}\right) \text { a.e. in } \Omega \text { and in } L^{2}(\Omega) \text { strongly. }
$$

Hence $\rho=p^{-1}\left(w_{\infty}+p_{\infty}\right)$ or $p(\rho)=A(\rho b)+p_{\infty}$. This is equivalent to

$$
\int_{\Omega}[\nabla p(\rho)-\rho b] \nabla \theta \mathrm{d} x=0 \quad \forall \quad \theta \in C_{0}^{\infty}(\Omega), \quad M \theta=0
$$

which yields

$$
\nabla p(\rho)=(I d-R)(\rho b)
$$


where $R$ is the projection of $L^{2}(\Omega)$ onto the closure in $L^{2}(\Omega)$ of the space of divergence free vector functions. Now, we want to prove that $R(\rho b)=0$. To this purpose let us take an arbitrary $\theta \in C_{0}^{\infty}(\Omega), \eta=R \theta$. Then we have

$$
\int_{\Omega} R(\widetilde{\rho}(s) b) \theta=\int_{\Omega}\left[\widetilde{\rho}(s) \frac{\partial v}{\partial s}+\widetilde{\rho}(s) v(s) \nabla v(s)-\operatorname{div} \tau_{i j}\right] \eta \mathrm{d} x .
$$

In the same way as before we can prove

$$
\left|\int_{t-1}^{t} \varphi(s-t) \int_{\Omega} R(\widetilde{\rho}(s) b) \theta \mathrm{d} x \mathrm{~d} s\right| \leq c\left\|\varphi^{\prime}\right\|_{L^{q}(0, T)}\|\eta\|_{W^{1,2}(\Omega)} \sigma(t)
$$

which implies that

$$
\left|\int_{\Omega} R(\widetilde{\rho}(t) b) \theta \mathrm{d} x\right| \leq\left\|\varphi^{\prime}\right\|_{L^{q}(0, T)}\|\eta\|_{W^{1,2}(\Omega)} \sigma(t), \quad t \geq 0 .
$$

Since $\|\eta\|_{W^{1,2}(\Omega)} \leq\|\theta\|_{W^{1,2}(\Omega)}$, we will obtain that

$$
R(\widetilde{\rho}(t) b) \rightarrow 0 \text { in } W^{-1,2}(\Omega) \text { strongly. }
$$

Then $R\left(\widetilde{\rho}\left(t_{n}\right) b\right) \rightarrow 0$ in $W^{-1,2}(\Omega)$ and $R\left(\widetilde{\rho}\left(t_{n}\right) b\right) \rightarrow R(\rho b)$ in $L^{2}(\Omega)$. Thus, $R(\rho b)=0$ and

$$
\nabla p(\rho)=\rho b \text { in } \Omega \text {. }
$$

Now, if $\pi$ is such a function that $\pi^{\prime}(r)=r^{-1} p^{\prime}(r)$ then, $\nabla \pi(\rho)=\nabla \xi$. Hence, we have $\pi(\rho)=g+h$ with some constant $h$ or $\rho=\pi^{-1}(g+h)$. It holds that

$$
\begin{aligned}
\int_{\Omega} \tilde{\rho}\left(x, t_{n}\right) \mathrm{d} x & =\int_{\Omega} \tilde{\rho}_{0} \mathrm{~d} x, \\
\int_{\Omega} \widetilde{\rho}(x) \mathrm{d} x & =\int_{\Omega} \tilde{\rho}_{0}(x) \mathrm{d} x .
\end{aligned}
$$

Since the function $a(h)=\int_{\Omega} \pi^{-1}(g+h) \mathrm{d} x$ is increasing, the function $\rho$ is uniquely determined by a unique constant $h^{*}$ satisfying $\int_{\Omega} \pi^{-1}\left(g+h^{*}\right) \mathrm{d} x=\int_{\Omega} \tilde{\rho}_{0} \mathrm{~d} x$. This implies

$$
\widetilde{\rho} \rightarrow \rho \text { in } L^{2}(\Omega) .
$$

We have proved the following result.

Theorem 3.1. Let $b_{i}=\frac{\partial \xi}{\partial x_{i}}, \xi \in W^{2, \infty}(\Omega), \xi$ small enough, $\frac{\mathrm{d} p}{\mathrm{~d} \rho}>0$ and $\mathcal{J}$ be the set of all solutions $(\widetilde{\rho}, \widetilde{u})$ of the problem (1.2)-(1.13) with the initial conditions $v_{0} \in W^{2, q}(\Omega) \cap W_{0}^{1,2}(\Omega), \rho_{0} \in C^{1}(\bar{\Omega})$. Then the rest state $(\rho, v)$ characterized by equations (1.1) is unconditionally asymptotically stable in the class $\mathcal{J}$ in the sense that there exists a subsequence $\left\{t_{n}\right\}, t_{n} \rightarrow+\infty$ such that

$$
\begin{aligned}
& \widetilde{\rho}\left(t_{n}\right) \rightarrow \rho \text { in } L^{2}(\Omega), \\
& \widetilde{v}\left(t_{n}\right) \rightarrow 0 \text { in } L^{2}(\Omega),
\end{aligned}
$$

and

$$
\nabla p(\rho)=\rho b \text { in } \Omega .
$$

Remark 3.1. The behaviour of incompressible Newtonian fluids under assumptions of sufficient regularity and $\rho>0$ was studied by Salvi and Straškraba [25]. Semigroup approach we can find in the work of Neustupa [21]. 
Acknowledgements. The present research of Š. Matušu-Nečasová has been supported under Grant No. 201/98/1450 of the Grant Agency of the Czech Republic. The research of M. Lukáčová-Medvidová has been supported by Grant No. 201/97/0153 and No. 201/00/0577 of the Grant Agency of the Czech Republic. The authors express their thanks for this support.

\section{REFERENCES}

[1] C. Amrouche and D. Cioranescu, On a class of fluids of grade 3, Laboratoire d'analyse numérique de l'université Pierre et Marie Curie, rapport 88006 (1988).

[2] C. Amrouche, Sur une classe de fluides non newtoniens : les solutions aqueuses de polymère, Quart. Appl. Math. L(4) (1992) 779-791.

[3] H. Bellout, F. Bloom and J. Nečas, Young measure-valued solutions for non-Newtonian incompressible fluids. Commun. Partial Differential Equations 19 (1994) 1763-1803.

[4] Beirão da Veiga, An $L^{p}$ - theory for the n-dimensional stationary compressible Navier-Stokes equations and the incompressible limit for compressible fluids. The equilibrium solutions. Comm. Math. Phys. 109 (1987) 229-248.

[5] D. Cioranescu and E.H. Quazar, Existence and uniqueness for fluids of second grade. Collège de France Seminars, Pitman Res. Notes Math. Ser. 109 (1984) 178-197.

[6] E. Feireisl and H. Petzeltová, On the steady state solutions to the Navier-Stokes equations of compressible flow. Manuscripta Math. 97 (1998) 109-116.

[7] E. Feireisl and H. Petzeltová, The zero - velocity limit solutions of the Navier-Stokes equations of compressible fluid revisited, in Proc. of Navier-Stokes equations and the Related Problem, (1999).

[8] G.P. Galdi, Mathematical theory of second grade fluids, Stability and Wave Propagation in Fluids, G.P. Galdi Ed., CISM Course and Lectures 344, Springer, New York (1995) 66-103.

[9] G.P. Galdi and A. Sequeira, Further existence results for classical solutions of the equations of a second grade fluid. Arch. Ration. Mech. Anal. 28 (1994) 297-321.

[10] D.D. Joseph, Fluid Dynamics of Viscoelastic Liquids. Springer Verlag, New York (1990)

[11] J. Málek, J. Nečas, M. Rokyta and R. Růžička, Weak and Measure-valued solutions to evolutionary partial differential equations. Chapman and Hall (1996).

[12] A.E. Mamontov, Global solvability of the multidimensional Navier-Stokes equations of a compressible fluid with nonlinear viscosity I. Siberian Math. J. 40 (1999) 351-362.

[13] A.E. Mamontov, Global solvability of the multidimensional Navier-Stokes equations of a compressible fluid with nonlinear viscosity II. Siberian Math. J. 40 (1999) 541-555.

[14] Š Matušù-Nečasová and M. Medvidová, Bipolar barotropic nonnewtonian fluid. Comment. Math. Univ. Carolin 35 (1994) $467-483$.

[15] S̆. Matušů-Nečasová, A. Sequeira and J.H. Videman, Existence of Classical solutions for compressible viscoelastic fluids of Oldroyd type past an obstacle. Math. Methods Appl. Sci. 22 (1999) 449-460.

[16] Š. Matušů-Nečasová and M. Medvidová-Lukáčová, Bipolar Isothermal non-Newtonian compressible fluids. J. Math. Anal. Appl. 225 (1998) 168-192.

[17] J. Nečas and M. Šilhavý, Multipolar viscous fluids. Quart. Appl. Math. XLIX (1991) 247-266.

[18] J. Nečas, A. Novotný and M. Šilhavý, Global solutions to the viscous compressible barotropic multipolar gas. Theoret. Comp. Fluid Dynamics 4 (1992) 1-11.

[19] J. Nečas, Theory of multipolar viscous fluids, in The Mathematics of Finite Elements and Applications VII MAFELAP 1990, J.R. Whitemann Ed., Academic Press, New York (1991) 233-244.

[20] J. Neustupa, A semigroup generated by the linearized Navier-Stokes equations for compressible fluid and its uniform growth bound in Hölder spaces, in Proc. of the International Conference on the Navier-Stokes equations, Theory and Numerical Methods, Varenna, June 1997, R. Salvi Ed., Pitman Res. Notes Math. Ser. 388 (1998) 86-100.

[21] J. Neustupa, The global existence of solutions to the equations of motion of a viscous gas with an artificial viscosity. Math. Methods Appl. Sci. 14 (1991) 93-119.

[22] J.G. Oldroyd, On the formulation of rheological equations of state. Proc. Roy. Soc. London A200 (1950) 523-541.

[23] K.R. Rajagopal, Mechanics of non-Newtonian fluids, in Recent Developments in Theoretical Fluid Mechanics Series 291, Longman Scientific \& Technical Reports (1993).

[24] M. Renardy, W.J. Hrusa and J.A. Nohel, Mathematical problems in Viscoelasticity, Longman, New York (1987).

[25] R. Salvi and I. Straškraba, Global existence for viscous compressible fluids and their behaviour as $t \rightarrow \infty$. J. Faculty Sci. Univ. Tokyo, Sect. I, A40 (1993) 17-51.

[26] W.R. Schowalter, Mechanics of Non-Newtonian Fluids. Pergamon Press, New York (1978).

[27] M.H. Sy, Contributions à l'etude mathématique des problèmes isssus de la mécanique des fluides viscoélastiques. Lois de comportement de type intégral ou différentiel. Thèse d'université de Paris-Sud, Orsay (1996).

[28] C. Truesdell and W. Noll, The Nonlinear Field Theories of Mechanics, 2nd edn. Springer, Berlin (1992). 